\title{
Conhecimento da população de Viçosa, M G, sobre as formas de transmissão da aids
}

\author{
The knowledge of the population of Viçosa, MG, \\ about the ways of AIDS transmission
}

\author{
M arcela M iranda Lima ${ }^{1}$ \\ Jaqueline Carlos ${ }^{1}$ \\ Rômulo Braga Areal ${ }^{1}$ \\ Rodrigo José Silva Souza ${ }^{1}$ \\ Swiany Silveira Lima \\ Lucio Antonio deOliveira Campos ${ }^{1}$ \\ Pedro Vasconcellos Eisenlohr ${ }^{1}$ \\ M ichellePicinin Filardi ${ }^{1}$
}

${ }^{1}$ Departamento de Biologia Geral, U niversidadeFederal deViçosa. Av. PH Rolfs s/n, Campus Universitário. 36570-000 Viçosa MG. limamm@gmail.com
Abstract AIDS is a major public health problem reaching all social strata. Knowing about theways of transmission is a way of preventing the disease. For evaluating the level of knowledge of the population of the city of Viçosa about AIDS transmission, a questionnaireabout the ways of transmission of the disease based on studies made in Europe was applied to 376 individuals. The questionnaire consisted of eleven multiple-choice questions about the ways of AIDS transmission, sex, age and educational level of the interviewees. The results showed that the ways of transmission emphasized in health campaigns are well assi milated, while the questions about everyday situations that offer no risk received a high number of incorrect answers. The differences were not significant when data were stratified by sex. The same data stratified by age or educational level showed significant differences for some questions; people aged over 55 and with a lower educational level had a significantly higher number of incorrect answers. It can be concluded that the population of $V$ içosa knows themain ways of AIDS transmission, but a part of the population does not know that some everyday situations do not pose risk of transmission. These data can be useful for the preparation of elucidative campaigns for re ducing prejudice.

Key words AIDS, Transmission, Knowledge, Educational level, Age
Resumo A aids éum dos mais graves problemas de saúde pública atuais e uma importante forma de prevenção reside no conhecimento, por parte da população, das formas detransmissão da doença. Com o objetivo de avaliar o grau de conhecimento da população da cidade deViçosa em relação às formas de transmi ssão da aids, foram aplicados 376 questionários, com onze perguntas de múltipla escolha sobre as formas de transmissão da aids, bem como sexo, idade e escolaridade dos entrevistados. Os dados mostraram queasformas de transmissão enfatizadas pelas campanhas de saúde estão bem assimiladas, enquanto situações do cotidiano que não oferecem risco apresentaram elevado número de respostas incorretas. As diferenças encontradas entre os sexos não foram significativas. Quando os dados foram estratificados por idade e escolaridade, foram encontradas diferençassignificativas para al gumas perguntas em que as pessoas com mais de 55 anos e de menor escolaridade apresentaram maior número de respostas incorretas. Pode-se concluir que a população vi çosense conhece as principais formas detransmi ssão da aids, masuma parte desconhece a ausência de risco de al gumas atividades cotidianas. Esses dados podem ser usados para a elaboração de campanhas de esclarecimento visando à redução do preconceito.

Palavras-chave Aids, Transmissão, Conhecimento, Escolaridade, Idade 
Introdução

A pandemia da aids (síndrome da imunodeficiência adquirida) caracteriza-se como um dos mais graves problemas de saúde pública deste início de século ${ }^{1}$. Dentre as doenças que mais matam no mundo, a aids perde apenas para doenças do coração e infecções respiratórias, sendo estas duasúltimas doenças que, tipicamente, causam morte em idade mais avançada². Sendo assim, a aids constitui-se hoje na maior causa de morte por doença entre o público jovem.

A aids já devastou vários países, revertendo quadros de desenvolvimento nacional, aumentando a distância entre ricos e pobres e empurrando grupos estigmatizados cada vez mais para a margem da sociedade². Milhões de pessoas já foram mortas, dizimando famílias e comunidades inteiras, e milhões de outras pessoas tiveram suas vidas negativamente afetadas pela doença ${ }^{3}$.

A aids foi descrita inicialmente em 1981, nos Estados Unidos, quando foram notificados os primeiros casos. Em 1983, foi identificado o agente etiológico, o vírus denominado HIV (vírus da imunodeficiência humana) ${ }^{4}$. Desdeentão, inúmeras têm sido as pesquisas em todos os campos associados à doença, como transmissão, prevenção e tratamento na tentativa do seu controle.

Deacordo com o Programa Nacional deDST e Aids do Ministério da Saúde, a epidemia da doença no Brasil está em processo de estabilização. Entre 1980 e 2004, foram registrados 362.364 casos no país. A incidência da aids diminuiu entre os homens, ao passo que aumentou entre as mulheres. O Brasil tem, hoje, aproximadamente 600 mil portadores do vírus HIV 5 . Segundo $\mathrm{M} \mathrm{elo}^{1}$, percebe-se queum quadro de uma epidemia restrita a determinados grupos de risco (como homossexuais, hemofílicos e usuários de drogas) foi substituído por outro no qual se destacam mulheres, jovens e heterossexuais.

NaXIII Conferêncialnternacional sobreAids, ocorrida em Durban, África do Sul, em 2000, cientistas chegaram a um consenso de que a meIhor estratégia para combater a aids seria integrando-se a prevenção com cuidados, tratamento e mitigação, sendo o programa brasileiro de combate à aids amplamente reconhecido como um exemplo dessa integração ${ }^{6}$.

Ainda não existem vacinas para a aids ${ }^{7} \mathrm{e}$, devido à ausência de cura até o presente momento, o controle da doença reside, hoje, na prevenção. Entretanto, o esforço para a prevenção da aids difere do de outros problemas de saúde, uma vez que as conseqüências de outros comportamen- tos de risco como fumo, bebida ou o uso de drogas são geralmente reversíveis, oferecendo assim múltiplas oportunidades de intervenção. Para muitos problemas de saúde, a falha em algum ponto da intervenção pode resultar ainda em sucesso posterior, mas a fal ha na intervenção no caso da aids pode ser a infecção pelo HIV e, na ausência de cura, quase certamente resultará em morte prematura ${ }^{8}$.

Fazem parte do processo preventivo: 0 de senvolvimento da intervenção, a sua implementação, disseminação, e por último, a avaliação dos programas 9 . A experiência ao longo dos vinte anos de epidemia mostrou que existem diversas alternativas de controle da disseminação da aids. Dentre estas alternativas, encontram-se campanhas de prevenção em massa realizadas na mídia; marketing social de preservativos (distribuição ou venda a baixo custo); tratamento de DSTs; projetos de prevenção realizados com públicos-alvo específicos como jovens, "garotas de programa" e seus clientes, usuários de drogas injetáveis; testagem eaconsel hamento voluntários e prevenção de transmissão mãe-filho. Estas diferentes formas têm se mostrado efetivas e, quando aplicadas e dosadas corretamente, podem levar à significativa redução nos níveis de prevalência da doença².

Independentemente do tipo de intervenção adotada, um efetivo planejamento eimplementação requerem um processo rigoroso de avaliações posteriores. No entanto, estas avaliações posteriores têm sido altamente negligenciadas na prática9. 0 M inistério da Saúde desenvolve inúmeras campanhas de massa visando à adoção de comportamentos seguros por parte da população, mas não há uma avaliação do resultado destas campanhas sobrea população. Para seter uma idéia, data de 2005 a primeira avaliação específica de uma campanha do M inistério da Saúde ${ }^{10}$.

Esta avaliação faz-se ainda mais necessária quando avaliados 0 alto custo das campanhas contra a aids e a escassez de recursos disponíveis. Assim, reveste-se de importância uma precisa e contínua avaliação do impacto de cada método de prevenção, para que uma análise custo/benefício seja feita, de modo que os investimentos possam ser feitos da melhor forma possível ${ }^{2}$.

As formas de avaliação dessas estratégias são na maioria das vezes específicas para cada uma, o que por vezes dificulta comparações. U ma forma de comparação geral e ampla é a avaliação do número de casos de aids detectados no período de aplicação da estratégia, por unidade detempo, em comparação com um período próximo 
sem aplicação na mesma população. O utra forma, mais simples, éa avaliação do conhecimento da população a respeito das questões abordadas nas campanhas, verificando diretamente $0 \mathrm{im}$ pacto das estratégias de informação².

0 presente trabalho teve como o objetivo avaliar o grau de informação da população da cidade de Viçosa acerca das formas de transmissão da aids, norteando, com os resultados obtidos, preparações de campanhas preventivas.

\section{Métodos}

Uma forma simples de se determinar a eficiência de programas de prevenção é a avaliação direta do conhecimento da população a respeito das questões abordadas nas campanhas². Sendo assim, para este estudo, adotou-se a aplicação de questionário estruturado, em virtude do seu baixo custo e por possibilitar respostas rápidas a questões relativas ao nível de conhecimento da comunidade.

\section{População de referência e amostra}

A cidade de Viçosa possuía, no último censo, 64.854 habitantes ${ }^{11}$, estando situada na Zona da $M$ ata sudeste do estado de M inas Gerais. A cidade abriga a Universidade Federal de Viçosa, tendo grande número de estudantes, professores e funcionários.

O questionário foi aplicado a transeuntes e trabalhadores da feira livre de agricultores do município, local este escolhido devido ao grande fluxo de pessoas de diferentes estratos populacionais. Os transeuntes foram abordados al eatoriamente, totalizando 376 questionários.

\section{Instrumento}

0 instrumento de coleta consistiu em um questionário com onze perguntas com respostas fechadas (sim, não, talvez e não sabe) e perguntas acerca de dados gerais (sexo, idade e grau de escolaridade). Como base para a elaboração do instrumento de coleta, foi utilizado um relatório de estudo realizado na União Européi $a^{12}$. Após a elaboração de uma primeira versão do questionário, este foi pré testado com dez pessoas, tendo sofrido algumas adaptações, sobretudo na linguagem utilizada.

Os procedimentos do estudo foram desenvolvidos de forma a proteger a privacidade dos indivíduos, garantindo a participação anônima e voluntária. 0 projeto de pesquisa incluindo 0 questionário foi avaliado e aprovado pelo ComitêdeÉtica em Pesquisa com seres humanos da Universidade Federal de Viçosa.

Coleta de dados

A equipedeentrevistadores foi composta por oito estudantes do curso de Ciências Biológicas da Universidade Federal de Viçosa entre 0 3o e 0 10 - período, participantes do Programa de Educação Tutorial (PET).

\section{Análise estatística}

A análise estatística dos dados foi realizada seguindo um critério de agrupamento de respostas, sendo as quatro possíveis respostas agrupadas em duas categorias, "informado" (inf) e "não informado" (n-inf).

A resposta "não" foi considerada informada para as questões: (1) Comendo alimentos preparados por uma pessoa com aids, (2) M anipulando objetos tocados por uma pessoa com aids, (3) Bebendo em um copo que acaba de ser usado por uma pessoa com aids, (6) U sando um vaso sanitário que acaba de ser utilizada por uma pessoa com aids, (7) Apertando a mão de uma pessoa com aids, (8) Beijando a boca de uma pessoa com aids, (10) Cuidando de uma pessoa com aids e (11) Doando sangue. As demais respostas (sim, talvez e não sabe) foram consideradas não informadas para estas mesmas questões.

A resposta "sim" foi considerada informada para as demais questões; (4) Recebendo sangue doado por uma pessoa com aids, (5) Sendo picado por uma seringa usada por uma pessoa com aids e (9) Tendo relações sexuais sem proteção com uma pessoa com aids. Os dados não agrupados em informado e não informado podem ser visualizados na Tabela 1.

As freqüências de respostas de informado e não informado referentes a cada pergunta foram então comparadas entre as variáveis sexo, idadee escolaridade, utilizando-se o teste do qui-quadrado ao nível de $5 \%$ de significância. Para os resultados significativos, foram testados os seguintes contrastes, utilizando-se o mesmo teste:

- M ais de 55 (+55) contra demais idade;

- Ensino fundamental contra ensino médio;

- Ensino fundamental e médio contra ensino superior. 
Resultados

Para o uso de seringas contaminadas, $97 \%$ dos entrevistados responderam tratar-se de uma for- ma de transmissão; para relação sexual sem proteção, 98\%; e recepção de sangue de doador contaminado, 97\%. A pergunta a respeito do aperto de mão teve $94 \%$ de respostas corretas (Figura 1).

Tabela 1. Resposta dos entrevistados às perguntas sobre situações que envolvem ou não risco de transmissão da aids.

\begin{tabular}{lrrrrrrrrrrr}
\hline Pergunta & 1 & 2 & 3 & 4 & 5 & 6 & 7 & 8 & 9 & 10 & 11 \\
\hline N ão & 291 & 288 & 251 & 9 & 5 & 227 & 326 & 150 & 6 & 299 & 237 \\
N ão sabe & 12 & 8 & 9 & 1 & 2 & 16 & 5 & 10 & 339 & 2 & 2 \\
Sim & 30 & 34 & 62 & 335 & 337 & 67 & 12 & 89 & 1 & 28 & 89 \\
Talvez & 13 & 16 & 24 & 1 & 2 & 36 & 3 & 97 & 0 & 17 & 18 \\
Total & 346 & 346 & 346 & 346 & 346 & 346 & 346 & 346 & 346 & 346 & 346 \\
\hline
\end{tabular}

Legenda: Respostas dos entrevistados para as respectivas perguntas sendo, $\mathrm{P} 1=$ Comendo alimentos preparados por uma pessoa com aids; $\mathrm{P} 2=\mathrm{M}$ anipulando objetos tocados por uma pessoa com aids; $\mathrm{P} 3=$ = Bebendo em um copo queacaba de ser usado por uma pessoa com aids; $\mathrm{P} 4=$ Recebendo sangue doado uma pessoa com aids; $\mathrm{P} 5=$ Sendo picado por uma seringa usada por uma pessoa com aids; $\mathrm{P} 6=\mathrm{U}$ sando um vaso sanitário que acaba de ser utilizada por uma pessoa com aids; $\mathrm{P} 7=\mathrm{A}$ pertando a mão de uma pessoa com aids; $\mathrm{P} 8=$ Beijando a boca de uma pessoa com aids; $\mathrm{P} 9=$ Tendo relações sexuais sem proteção com uma pessoa com aids; P10= Cuidando de um uma pessoa com aids; $P 11=$ D oando sangue.

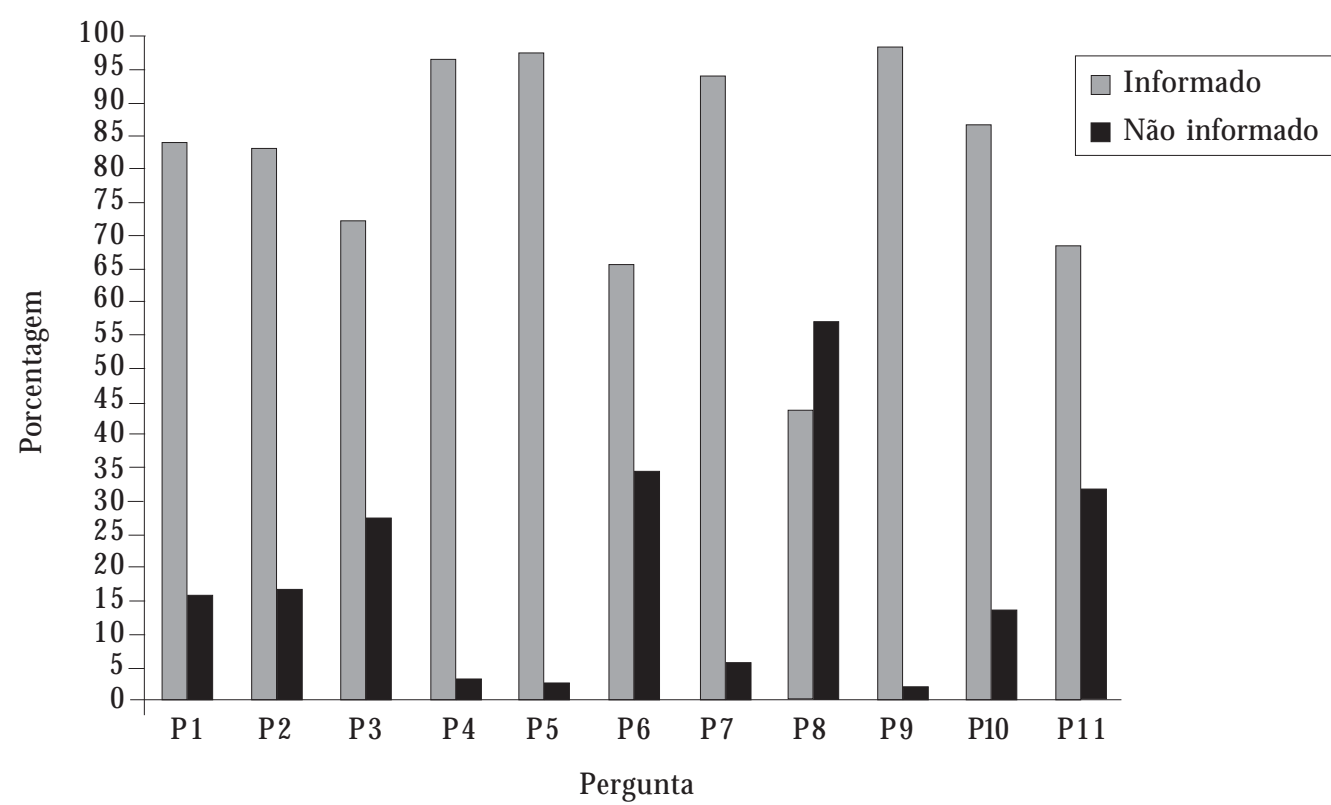

Legenda: Porcentagens das respostas agrupadas em informado enão informado para as seguintes perguntas: $\mathrm{P} 1=\mathrm{C}$ omendo alimentos preparados por uma pessoa com aids; $\mathrm{P} 2=\mathrm{M}$ anipulando objetos tocados por uma pessoa com aids; $\mathrm{P} 3=\mathrm{Bebendo}$ em um copo que acaba de ser usado por uma pessoa com aids; $\mathrm{P} 4=$ Recebendo san que doado uma pessoa com aids; $\mathrm{P} 5=$ Sendo picado por uma seringa usada por uma pessoa com aids; $\mathrm{P} 6=\mathrm{U}$ sando um vaso sanitário que acaba de ser utilizada por uma pessoa com aids; $\mathrm{P7}=$ Apertando a mão de uma pessoa com aids; $\mathrm{P} 8=$ Beijando a boca de uma pessoa com aids; $\mathrm{P} 9=$ Tendo relações sexuais sem proteção com uma pessoa com aids; $\mathrm{P} 10=$ Cuidando de um uma pessoa com aids; $\mathrm{P} 11=$ D oando sangue.

Figura 1. Freqüências e porcentagens das respostas para as respectivas perguntas. 
Quanto a outras possíveis formas de transmissão, $73 \%$ dos entrevistados responderam que não se pode contrair o vírus ao beber em um copo que acaba de ser usado por uma pessoa com aids, $66 \%$ ao usar um assento sanitário nestas condições, $43 \%$ beijando a boca de um portador e $68 \%$ ao doar sangue simplesmente.

Quando os dados foram estratificados por sexo, apenas a pergunta referente à transmissão por beijo na boca teve diferença significativa, sendo que as mulheres apresentaram maior número derespostas corretas $\left(\chi^{2}=5.82 ; p=0.01\right)$. Quando os mesmos foram estratificados por idade menores e maiores que 55 anos - , foram encontradas diferenças para as perguntas 1, 2, 3, 6, 7, 8 e 10 (Tabela 2), em que as pessoas com mais de 55 anos apresentaram menor número de respostas corretas. $\mathrm{Na}$ estratificação por grau de escolaridade, houve diferença entre as pessoas que possuíam atéo ensino fundamental eaquelas que possuíam ensino médio completo para as perguntas 1, 2, 3, 6, 8 e 10 (Tabela 3), em queaquelas que possuíam o ensino médio tiveram maior número de respostas corretas do que aquelas que possuíam até 0 ensino fundamental. Também houve diferença entre as pessoas que possuíam até 0 ensino médio e aquelas que possuíam ensino superior para as perguntas 1, 3, 6, 8 e 11 ( Tabela 3), para as quais as pessoas com nível supe rior tiveram maior número de acertos.

\section{Discussão}

Na primeira avaliação de Campanha de Saúde realizada pelo Ministério da Saúde, Porto ${ }^{10}$ encontrou um resultado que demonstra sucesso de campanha vinculada pela mídia, em especial a televisão. Os dados do presente estudo mostram que as formas de transmissão como a transmissão sexual, o uso de seringas contaminadas e a recepção de sangue de doador contaminado, enfatizadas pelas campanhas de saúde e pela mídia em geral, estão bem sedimentadas (Figura 1). 0 aperto de mão, apesar de não ser tão enfatizado pela mídia, teve um alto número de respostas corretas.

Entretanto, caberessaltar queo conhecimento da população sobre as formas de transmissão de doenças (neste caso, a aids) não implica necessariamente em mudança de atitude ${ }^{13,14}$. Esteé um grande desafio na elaboração de campanhas educativas, uma vez que além de chegar até a população, a mensagem vinculada deve ser capaz de provocar mudanças comportamentais. Segundo $\mathrm{Chor}^{14}$, ao pensarmos na prevenção de doenças, são necessárias estratégias adequadas a realidades específicas. Em 2003, a campanha de carnaval de prevenção à aids do Ministério da Saúde, por exemplo, foi voltada a um público específico - meninas entre 13 e 19 anos, mostrando-Ihes a necessidade de que elas não tivessem

Tabela 2. Comparação percentual de respostas corretas entre dois grupos de idade (maiores e menores de 55 anos).

\begin{tabular}{|c|c|c|c|c|}
\hline Pergunta & $\begin{array}{l}-55 \text { anos } \\
(n=306)\end{array}$ & $\begin{array}{c}+55 \text { anos } \\
(n=40)\end{array}$ & $\chi^{2}$ & $\mathrm{p}$ \\
\hline 1.Comendo alimentos preparados por uma pessoa com aids & $86.3 \%$ & $67.5 \%$ & 9.327 & $<0.01 *$ \\
\hline 2. Manipulando objetos tocados por uma pessoa com aids & $85.6 \%$ & $65.0 \%$ & 10.781 & $<0.01 *$ \\
\hline 3. Bebendo em um copo que acaba de ser usado por uma pessoa com aids & $76.1 \%$ & $45.0 \%$ & 17.227 & $<0.01 *$ \\
\hline 4. Recebendo sangue doado uma pessoa com aids & $96.4 \%$ & $100.0 \%$ & - & - \\
\hline 5. Sendo picado por uma seringa usada por uma pessoa com aids & $97.4 \%$ & $97.5 \%$ & 0.002 & 0,966 \\
\hline 6. U sando um vaso sanitário que acaba de ser utilizada por uma pessoa com aids & $69.3 \%$ & $37.5 \%$ & 15.835 & $<0.01 *$ \\
\hline 7. Apertando a mão de uma pessoa com aids & $96.1 \%$ & $80.0 \%$ & 16.792 & $<0.01 *$ \\
\hline 8. Beijando a boca de uma pessoa com aids & $76.1 \%$ & $35.0 \%$ & 29.318 & $<0.01 *$ \\
\hline 9. Tendo relações sexuais sem proteção com uma pessoa com aids & $97.7 \%$ & $100.0 \%$ & - & - \\
\hline 10. Cuidando de um uma pessoa com aids & $89.2 \%$ & $65.0 \%$ & 17.672 & $<0.01^{*}$ \\
\hline 11. Doando sangue & $68.6 \%$ & $67.5 \%$ & - & - \\
\hline
\end{tabular}

Legenda: * Diferenças probabilísticas significativas a $5 \%$ pelo teste qui-quadrado

Os valores de p ausentes referem-se a contrastes não realizados, pois não houve diferença entrenenhuma faixa etária. 
Tabela 3. Comparação percentual de respostas corretas entre três grupos de escolaridade.

\begin{tabular}{|c|c|c|c|c|c|c|c|c|}
\hline Pergunta & $\begin{array}{c}\text { AtéEF } \\
(n=194)\end{array}$ & $\begin{array}{c}\text { EM } \\
(n=104)\end{array}$ & $\chi^{2}$ & $p$ & $\begin{array}{l}\text { Até EM } \\
(n=298)\end{array}$ & $\begin{array}{c}\text { ES } \\
(n=48)\end{array}$ & $\chi^{2}$ & $p$ \\
\hline $\begin{array}{l}\text { 1. Comendo alimentos preparados por } \\
\text { uma pessoa com aids }\end{array}$ & $78.3 \%$ & $89.4 \%$ & 5.677 & $0,017 *$ & $82.2 \%$ & $95.8 \%$ & 5.735 & $0,017 *$ \\
\hline $\begin{array}{l}\text { 2. M anipulando objetos tocados por uma } \\
\text { pessoa com aids }\end{array}$ & $78.3 \%$ & $90.4 \%$ & 6.807 & $<0.01 *$ & $82.5 \%$ & $87.5 \%$ & 0.726 & 0,394 \\
\hline $\begin{array}{l}\text { 3. Bebendo em um copo que acaba de ser } \\
\text { usado por uma pessoa com aids }\end{array}$ & $63.9 \%$ & $80.8 \%$ & 9.121 & $<0.01^{*}$ & $69.8 \%$ & $89.6 \%$ & 8.124 & $<0.01 *$ \\
\hline $\begin{array}{l}\text { 4. Recebendo sangue doado uma pessoa } \\
\text { com aids }\end{array}$ & - & - & - & - & - & - & - & - \\
\hline $\begin{array}{l}\text { 5. Sendo picado por uma seringa usada } \\
\text { por uma pessoa com aids }\end{array}$ & - & - & - & - & - & - & - & - \\
\hline $\begin{array}{l}\text { 6. U sando um vaso sanitário que acaba de } \\
\text { ser utilizada por uma pessoa com aids }\end{array}$ & $56.2 \%$ & $75.0 \%$ & 10.253 & $<0.01 *$ & $62.7 \%$ & $83.3 \%$ & 7.761 & $<0.01 *$ \\
\hline $\begin{array}{l}\text { 7. Apertando a mão de uma pessoa com } \\
\text { aids }\end{array}$ & - & - & - & - & - & - & - & - \\
\hline $\begin{array}{l}\text { 8. Beijando a boca de uma pessoa com } \\
\text { aids }\end{array}$ & $63.4 \%$ & $78.8 \%$ & 7.522 & $<0.01^{*}$ & $68.8 \%$ & $87.5 \%$ & 7.084 & $<0.01^{*}$ \\
\hline $\begin{array}{l}\text { 9. Tendo relações sexuais sem proteção } \\
\text { com uma pessoa com aids }\end{array}$ & - & - & - & - & - & - & - & - \\
\hline $\begin{array}{l}\text { 10. Cuidando de um uma pessoa com } \\
\text { aids }\end{array}$ & $81.4 \%$ & $93.3 \%$ & 7.669 & $<0.01^{*}$ & $85.6 \%$ & $91.7 \%$ & 1.309 & 0,253 \\
\hline 11. Doando sangue & $63.9 \%$ & $67.3 \%$ & 0.342 & 0,558 & $65.1 \%$ & $89.6 \%$ & 11.484 & $<0.01^{*}$ \\
\hline
\end{tabular}

Legenda: $\mathrm{EF}=$ Ensino fundamental; $\mathrm{EM}=\mathrm{Ensino}$ médio; $\mathrm{ES}=$ Ensino superior

$*$ Diferenças probabilísticas significativas a $5 \%$ pelo teste qui-quadrado

Os valores dep ausentes referem-se a contrastes não realizados, pois não houve diferença entrenenhum grupo de escolaridade.

vergonha deadquirir preservativos. Em uma pesquisa realizada para avaliar os efei tos dessa campanha, descobriu-sequeela foi bem recebida entre as jovens, além de ter causado discussões sobreo tema, e aparentemente ter contribuído para reforçar atitudes ${ }^{10}$.

Um fato importante observado em nosso estudo merece especial atenção: o conhecimento equivocado quanto a determinadas formas de transmissão, como 0 uso de assento sanitário, 0 beijo na boca, a doação desanguee o uso de copo previamente utilizado por um portador da doença. Essas questões apresentaram os menores níveis de acerto, todos inferiores a $75 \%$. Resultados semelhantes também foram observados em outros estudos ${ }^{13}$. As respostas erradas obtidas podem ser decorrentes das dimensões culturais ou simbólicas da aids, incluídas no imaginário do risco da doença ${ }^{15}$. 0 imaginário do risco da aids relaciona-se com os significados atribuídos à doença de maneira negativa, uma vez que esta há muito tempo vem sendo atribuída à idéia decontágio, à necessidade de limites corporais e à simbologia do sangue e saliva ${ }^{15}$. Este imaginário e simbolismo geram, em muitos casos, a falta de informação da população que associa a possibilidade de contaminação a diversas situações do cotidiano que não oferecem risco.

Quanto às perguntas sobre outras situações do cotidiano que não oferecem risco de transmissão, como comer alimentos preparados por um doente, manipular objetos tocados por estee cuidar de um portador da doença, os índices de acerto situaram-se entre 75 e 90\%. Embora estas situações também recebam pouca atenção da mídia, elas apresentaram um índice de acerto maior do que as acima citadas. Este resultado podeser explicado por constituírem situaçõesque não envolvem os grandes símbolos associados à doença: sangue e saliva.

As respostas relacionadas à pergunta referente ao beijo na boca chamam bastante atenção devido ao grande número de pessoas que acreditam que este oferece algum risco (57\%). A resposta "talvez", que foi considerada incorreta, representou $28 \%$ do total, refletindo a falta de consenso existentena comunidadecientífica. $N$ a dé cada de 1980, muitas informações contraditórias foram vinculadas em relação à transmissão do vírus através do beijo na boca ${ }^{13}$ eeste conceito 
pode ter sido incorporado pelas pessoas e influenciado suas respostas. Embora o M inistério da Saúde ${ }^{16}$ desconsidere o beijo na boca como forma de contaminação e pesquisadores afirmem que a saliva não contém vírus em quantidades infectantes ${ }^{17}$, grande parte da população ainda apresenta dúvida quanto a esta situação. Fernandes et al. ${ }^{13}$, em sua pesquisa em uma população de favela do Rio de Janeiro, encontraram 27,1\% de respostas incorretas, número bastante semeIhanteao encontrado nestetrabalho, catorzeanos depois, indicando uma falha na divulgação desta informação.

Embora, como visto acima, a maioria da população esteja consciente de que a recepção de sangue contaminado é uma forma de transmissão, muitos entrevistados acreditam que a doação de sangue traga algum tipo de risco para o doador (32\%). Dados semelhantes foram encontrados por Sawyer et al. ${ }^{18}$, em que pessoas entrevistadas responderam que há risco de contaminação no ato de doação de sangue. Estes dados podem refletir a falta de campanhas educativas de hemocentros, esclarecendo a população sobre as medidas de prevenção tomadas para que o doador não corra nenhum risco. Doadores e receptores estão protegidos pela legislação ${ }^{19}$, onde o uso de material descartável em todas as fases do processo e o teste do sangue a ser utilizado é obrigatório. Percebeu-se uma confusão dos entrevistados entre 0 ato de doar e 0 de receber sangue, sendo que, para muitos, doação correspondia ao ato de receber sangue via transfusão, o que pode justificar parte dos resultados encontrados. Este tipo de dúvida pode acarretar uma menor procura por doação de sangue, o quenão se é desejável para a saúde pública.

O fragmento a seguir foi extraído da cartilha da campanha do Dia M undial de Luta contra a Aids do Governo Federal ${ }^{20}$ e aponta para o problema da falta de informação da população em geral: 0 preconceito e a discriminação contra as pessoas vivendo com HIV/aids são as mai ores barreiras no combateà epidemia, ao adequado apoio, à assistência eao tratamento da aids eao seu diagnóstico. Os estigmas são desencadeados por motivos que incluem a falta de conhecimento, mitos e medos. A pesar de haver essa consciência, as campanhas ainda são fortemente voltadas para as formas de transmissão, dando menor enfoque às formas de não transmissão.

Quando os dados foram estratificados por idade (menor e maior que 55 anos), observou-se um número significativamente maior de respostas incorretas para os maiores de 55 anos justa- mente nas questões pouco abordadas pela mídia nas campanhas. Cabe ressaltar que, para as formas de transmissão bem enfocadas pela mídia, não houve diferença entreas idades, podendo este fato estar também associado às informações obtidas na escola, relacionadas às formas de nãotransmissão. Este resultado pode ser reflexo de campanhas informativas destinadas a um público mais jovem eà resistência ou fal ta deacesso do público mais velho aos novos conhecimentos a respeito da doença. Há ainda o fato de que, no início da epidemia da aids, a doença era associada à idéia de morte, e as campanhas visando à prevenção da doença estiveram amplamente associadas ao terrorismo, ao medo ${ }^{21}$. Segundo Ayres ${ }^{21}$, estas campanhas terroristas tiveram um efeito de aumento da discriminação e do preconceito com relação à doença, além de afastar as pessoas do problemanaépoca. 0 elevado índice de respostas erradas encontrados nesta faixa etária para situações de não-transmissão pode contribuir grandemente para o aumento do preconceito com relação aos portadores da doença. Campanhas direcionadas a indivíduos acima de 55 anos, como em grupos de terceira idade, e campanhas veiculadas pela televisão abordando as formas de nãotransmissão podem ser uma boa opção para reverter este quadro.

$\mathrm{Na}$ estratificação por escolaridade, indivíduos que possuíam ensino superior apresentaram maior número de respostas corretas com relação a indivíduos com ensino médio, e estes, por sua vez, apresentaram maior índice de acerto quando comparados com aqueles que possuíam até 0 ensino fundamental. Com estes dados, pode-se dizer que, para a população amostrada neste estudo, a escolaridade está diretamente relacionada ao conhecimento sobre as formas de transmissão e não-transmissão da doença. Questões envolvendo as situações de não-transmissão, como preparo de alimento, uso de copo, uso de vaso sanitário e beijo foram aquelas cujas respostas estiveram mais relacionadas com a escolaridade, ocorrendo um aumento gradativo no número de respostas corretas com maiores níveis de escolaridade. Por outro lado, questões envolvendo situações de transmissão tiveram número de acertos independentes da escolaridade. A doação de sangue mais uma vez chama a atenção pelos resultados encontrados. Não foi verificada diferença significativa entreensino fundamental e médio (cerca de 35\% de erro para ambos), contrastando com um menor número de respostas erradas entre os que possuem ensino superior ( $10 \%$ de erro). 
Segundo Pitta ${ }^{22}$, a questão comunicacional no campo da saúde passa a não mais se reduzir a planejamento e elaboração de produtos comunicativos, mas a se conformar como um conjunto mais complexo de processos, estratégias, táticas einventividades, entre as quais os produtos comunicativos são apenas parte. Assim, a campanha em si éo resultado final de todo uma estratégia de ação com vistas à saúde da população. Entretanto, para se montar uma estratégia, é preciso primeiro conhecer o problema, ou seja, conversar com a população, saber o que ela já sabe, se os meios atualmente utilizados são eficientes, se são aprovados, enfim, é preciso um envolvimento dos sujeitos com o problema a ser trabalhado.

\section{Conclusão}

Pode-seconcluir queas pessoas têm conhecimento sobre as principais formas de transmissão da aids, mas não se encontram bem esclarecidas quanto a situações do cotidiano que não oferecem risco.

Os dados obtidos poderão servir de basepara futuras campanhas sobre a aids, na qual o enfoqueseja o esclarecimento da população quanto a situações que não oferecem risco, visando à redução do preconceito e da discriminação contra as pessoas com aids.

Anexo. QUESTIONÁRIO APLICADO

Sexo: $F$ M

\begin{tabular}{lllllll}
\hline Faixa etária: & $0-15$ & $15-25$ & $25-35$ & $35-45$ & $45-55$ & 55 em diante \\
\hline Escolaridade: & Até EF & EM & ES & & \\
& & & & & \\
\hline
\end{tabular}

\begin{tabular}{|c|c|c|c|c|c|}
\hline & A aids pode ser pega por quais das seguintes maneiras? & Sim & Não & Talvez & Não sabe \\
\hline 1 & Comendo alimentos preparados por doente ou portador da aids & & & & \\
\hline 2 & M anipulando objetos tocados por doente ou portador da aids & & & & \\
\hline 3 & $\begin{array}{l}\text { Bebendo em um copo que acaba de ser usado por doente ou portador } \\
\text { da aids }\end{array}$ & & & & \\
\hline 4 & Recebendo sangue doado por um doente ou portador da aids & & & & \\
\hline 5 & Sendo picado por uma seringa usada por doente ou portador da aids & & & & \\
\hline 6 & $\begin{array}{l}\text { Usando um vaso sanitário que acaba de ser utilizado por um doente } \\
\text { ou portador da aids }\end{array}$ & & & & \\
\hline 7 & Apertando a mão de um doente ou portador da aids & & & & \\
\hline 8 & Beijando a boca de um doente ou portador da aids & & & & \\
\hline 9 & $\begin{array}{l}\text { Tendo relações sexuais sem proteção com um doente ou portador da } \\
\text { aids }\end{array}$ & & & & \\
\hline 10 & Cuidando de um doente ou portador da aids & & & & \\
\hline 11 & Doando sangue & & & & \\
\hline
\end{tabular}




\section{Colaboradores}

Todos os autores participaram da concepção e do delineamento do trabalho, bem como na coleta dos dados (aplicação dos questionários). RB Areal trabal hou na análise dos dados, M M Lima, na confecção dos gráficos e tabelas e todos os autores, na interpretação dos mesmos. M M Lima, J Carlos, RB Areal, RJS Souza, SS Lima, M P Filardi e PV Eisenlohr participaram da redação inicial do artigo eLAO Campos fez a revisão do mesmo. M M Lima eJ Carlos foram responsáveis pela redação final do artigo.

\section{Agradecimentos}

Agradecemos à N erilda M artins M iranda de Lima, atual coordenadora do Serviço de Saúde do Município de Viçosa, que nos incentivou a realizar este trabalho; ao Odair Correia Campos, pelo auxílio na coleta dos dados; ao Ronaldo Reis Júnior, Prof. Paulo de M arco Júnior e Flávia de Lima pelo auxílio nas análises estatísticas. Agradecemostambém ao Programa de Educação Tutorial, mantido pela SESU-M EC, pelo auxílio financeiro.

\section{Referências}

1. M elo MGBA. Perfil epidemiológico dos portadores HIV/aids atendidos no centro de referência de DST/ HIV/aids no município de Sobral - CE [monografia]. Sobral (CE): Universidade Estadual Vale do Acaraju; 2004.

2. Walker D. Cost and cost-effectiveness of HIV/aids prevention strategies in developing countries: is there an evidence base? Health Policy Plan 2003; 18(1):4-17.

3. Lamptey PR. Reducing heterosexual transmission of HIV in poor countries. Br M ed J 2002; 324: 207-211.

4. Rachid M, Shechter M. M anual de HIV/aids. Rio de Janeiro: Revinter; 2000.

5. Brasil. M inistério da Saúde. Programa Nacional de DST e aids 2005. [acessado 2006 Jan]. Disponível em: http://www.aids.gov.br

6. Berkman A, Garcia J, Muñoz-Laboy M, Paiva V, Parker R. A Critical Analysis of the Brazilian Response to HIV/aids: Lessons Learned for ControIling and Mitigating the Epidemic in Developing Countries. Am J Public Health 2005; 7:1162-1172.

7. Lemckert AAC, Goudsmit J, Barouch DH. Challenges in the search for an HIV vaccine. Eur J Epidemiol 2004; 19(6):513-516.

8. Chesney MA. Prevention of HIV and STD infections. Prev Med 1994; 23:655-660.

9. Kelly JA, M urphy DA, Sikkema KJ, Kalichman SC. Psychological interventions to prevent HIV infection are urgently needed. Am Psychol 1993; 48:10231034.

10. Porto MP. Lutando contra a aids em meninas adolescentes: os efeitos da Campanha de Carnaval do M inistério da Saúde do Brasil. Cad Saúde Pública 2005; 21(4):1234-1243.

11. IBGE. Censo Demográfico 2000 - Resultados do universo. [acessado $2006 \mathrm{M}$ ar]. Disponível em: http:// www.ibge.gov.br/home/estatistica/populacao/censo $2000 /$ universo $\cdot$ php?tipo $=31 \&$ paginaatual $=1 \&$ uf $=$ $31 \&$ letra $=\mathrm{V}$

12. European Opinion Research Group. Le SIDA. Eurobarometre Special 2003; 183(2):1-15.

13. Fernandes JCL, Coutinho ESF, M atida A. Conhecimentos e atitudes relativas à SIDA/aids em uma população de favela do Rio de Janeiro. Cad Saúde Pública 1992; 8(2): 176-182.

14. Chor D. Saúde pública e mudanças de comportamento: uma questão contemporânea. Cad Saúde Pública 1999; 15(2):423-425.

15. Jeolás L. Os jovens e o imaginário da aids: notas para uma construção social do risco. Campos Revista de Antropologia Social [periódico na Internet] 2003 [acessado $2006 \mathrm{M}$ arço]; 4: [cerca de 19 p.] Disponível em: http://calvados.c3sl.ufpr.br/ojs2/ index.php/campos/article/viewFile/1600/1348

16. Brasil. M inistério da Saúde. Controle de infecções e a prática odontológica em tempos de aids: manual de condutas. Brasília: Coordenação Nacional de DSTAids/ Secretaria de Políticas de Saúde/M inistério da Saúde; 2000.

17. Machado ARL, Silva CLO, Dutra CE, Galvão NAM . Aids na infância: orientação básica no atendimento. J Pediatr (Rio J) 1994; 1(70):5-9. 
18. Sawyer D, Figoli MGB, Rodrigues RN, Garcia RA. Caracterização dos tipos de doadores de sangue em Belo Horizonte: heterogeneidade do homogêneo. XII Encontro de Estudos Populacionais; 2000; Caxambu. p. 1-21.

19. Lei Estadual n 13.317, Capítulo VII, Art. 72 de 24 de setembro de 1999. Código de Saúde do Estado de M inas Gerais. M inas Gerais Diário do Executivo 1999; 25 set.

20. Brasil. Ministério da Saúde. Programa Nacional de DST e Aids 2002. [acessado 2006 Jan]. Disponível em: http://www.aids.gov.br/final/dia_2002/ dia_mundial.htm

21. Ayres JRCM. Práticas educativas e prevenção de HIV/aids: lições aprendidas e desafios atuais. Interface - Comunicação, Saúde, Educação [periódico na Internet] 2002 [acessado $2007 \mathrm{M}$ ar]; 6(11):1[cerca de 23 p.]. Disponível em: http://www.interface. org.br/revistall/ensaiol.pdf

22. Pitta AM, Rivera FJU. Sobre pontos de partida: planejamento em comunicação e integralidade da atenção em saúde. Interface - Comunicação, Saúde, Educação [periódico na Internet] 2006 [acessado $2007 \mathrm{Mar}$ ]; 10(20):[cerca de 11 p.]. Disponível em: http://www.scielo.br/pdf/icse/v10n20/09.pdf

Artigo apresentado em 07/04/2006

Aprovado em 02/03/2007 